\title{
Celebrating 200 issues of Bioanalysis
}

\author{
Sankeetha Nadarajah*,1 \\ ${ }^{1}$ Future Science Group, Unitec House, 2 Albert Place, London N3 1QB, UK \\ *Author for correspondence: s.nadarajah@future-science.com
}

First draft submitted: 4 April 2018; Accepted for publication: 6 April 2018; Published online: 14 June 2018

Keywords: analytical chemistry • Bioanalysis • publications • research

Welcome to the $200^{\text {th }}$ issue of Bioanalysis. Focusing on the techniques and methods used for the detection or quantitative study of analytes in biological samples, the journal brings cutting-edge technologies, advancements and perspectives to its audience, encouraging dialogue and debate among the bioanalytical community.

Since the launch of the journal in 2009 we have evolved steadily, as has the field of bioanalysis, adapting to the needs of the time from both a bioanalytical and publishing perspective, ensuring we publish quality content in timely and accessible formats for the modern bioanalyst. This quest led to the launch of our sister website Bioanalysis Zone in 2011 - an interactive online resource for the bioanalytical community [1].

Having worked on the journal since 2010 across the Commissioning, Production and Digital departments, I've personally witnessed the inception and development of ideas through working closely with experts and our dedicated editorial board, the enthusiasm of contributors in the preparation and finalization of quality content, and also the general openness of the field to feedback from peers and editorial staff; understanding the need to maintain and push for higher standards at every stage. We are confident we'll see this trend continuing, over upcoming years.

In this article, we would like to take a look back a handful of the most-read content published in Bioanalysis.

\section{Assessing the matrix effects of hemolyzed samples in bioanalysis (2009)}

The validation of LC-MS/MS assays includes an assessment of the matrix effect, which is the direct or indirect alteration or interference in the instrumental response (including sample preparation) due to the presence of matrix constituents in extracted samples [2]. The hemolysis effect is a type of matrix effect that can also have an impact on analyte quantitation, rendering the analytes immeasurable in some cases. In this Challenge article, Hughes et al. reported examples from four bioanalytical methods, where the presence of hemolyzed blood in plasma was found to have an impact on analyte quantitation, going on to provide a description of the solutions adopted to resolve this bioanalytical challenge [3].

\section{Nicotine exposure \& metabolizer phenotypes from analysis of urinary nicotine \& its 15 metabolites by LC-MS (2011)}

In order to rigorously assess tobacco smoke exposure in smokers, it is essential to have an accurate method for quantifying nicotine and its known metabolites. In this Research article, Rangiah et al. developed a stable-isotope dilution LC-MRM/MS assay for quantification of urinary nicotine and the 15 possible metabolites that could arise from known metabolic pathways. The idea was to allow the determination of whether there are relationships between nicotine exposure and/or metabolizer phenotype with exposure to toxic substances present in tobacco smoke and/or to biological response biomarkers to tobacco smoking [4].

\section{Validation \& verification of measurement methods in clinical chemistry (2012)}

This Review article by Theodorsson presented an overview of validation and verification procedures in clinical chemistry, focusing on the use of harmonized concepts and nomenclature, fitness-for-purpose evaluations and procedures for minimizing overall measurement and diagnostic uncertainty. The need for mutually accepted validation procedures in all fields of bioanalysis as in 2012, remains a continuous quest as we have especially witnessed from discussions and debates at bioanalytical conferences across the globe. The author acknowledged that "internationally agreed measurement units and nomenclature are fully implemented only painfully slowly... The 
entire world is already extensively technically and economically integrated and in need of further armonization in the field of bioanalysis. Hopefully, such extensive harmonization is not more than a generation away" [5].

\section{Interference in immunoassays to support therapeutic antibody development in preclinical \& clinical studies (2014)}

Immunoassays are used to measure the concentration of the therapeutic antibody, anti-drug antibodies and soluble protein biomarkers during preclinical and clinical studies. The reliability of these assays is crucial as the results are used for safety assessment and dose selection. This Perspective article by Schwickart et al. discusses the source of interference and strategies to mitigate or eliminate interference in immunoassays used during preclinical and clinical drug development of drugs, with a focus on the development of therapeutic antibodies [6].

\section{Issues facing the bioanalytical community: summary of round table discussions (2016)}

As mentioned earlier, Bioanalysis and Bioanalysis Zone work in collaboration on several projects, with the vision of ensuring research and advancements in the field are presented in the most appropriate manner to the bioanalytical community. One of the successful collaborations we have worked on together is the organization of Panel Discussions in which experts from leading CROs, consultancies and pharmaceutical companies converse about overarching and timely themes from the industry. The first roundtable discussion was held in Orlando, USA in April 2016 and focused on 'Issues facing the bioanalytical community'. The panel was chaired by Neil Spooner (Spooner Bioanalytical Solutions), the Senior Editor of the journal [7], and was the first of many such discussions we have hosted, and published the subsequent discussion reports on. We hope these continue to encourage dialogue and debate on timely topics within the community.

\section{Rapid prototyping using 3D printing in bioanalytical research (2017)}

As well as our longer Research and Review-style content, our short editorial pieces providing a snapshot of issues of topical importance to the bioanalytical community often create an imprint on niche issues. This Editorial by Zhang et al. discussed how in bioanalytical research laboratories, 3D printing was no longer just a concept; but had become a useful tool for the fabrication of various analytical devices and custom labware over the last few years. They looked at some of examples from their lab as well as those from others. They concluded "...3D printing has the potential to be utilized in more chemical and biological applications and change the perceived limitations in the experimental design for bioanalytical studies" [8].

\section{The latest content}

We've already had a great response (in terms of downloads and number of times accessed) to some of our more recent publications, including Feedback from the European Bioanalysis Forum: focus workshop on current analysis of immunogenicity: best practices and regulatory hurdles' [9]. Our meeting reports often feature in our top read content and we endeavour to cover as many symposiums as possible to provide our readers with a summary of the key discussions occurring at these important gatherings of experts, often from around the globe.

We have also recently published a special feature on The Decennial Index of the White Papers in Bioanalysis: A Decade of Recommendations (2007-2016)' [10]. After 10 years of publishing White Papers summarizing the conclusions and consensus points from each Workshop on Recent Issues in Bioanalysis (WRIB) meeting, in which a wide range of industry opinion leaders and regulatory authorities come together, the recent publication acts as a general index organized by topic to easily consult all these recommendations and their evolution over time. Watch out for this year's White Papers in the November and December issues.

Bioanalysis has worked closely with several such organizations over the years, publishing widely discussed publications. These include, for example, White Papers from the WRIB workshops and the European Bioanalysis Forum, as well as Conference Reports from meetings such as the Japanese Bioanalysis Forum Symposium, China Bioanalysis Forum meeting, CPSA symposiums, Land O'Lakes Bioanalytical Conference and the GCC closed forum. We very much look forward to further establishing our working relationship with these organizations and making new connections in the future.

The Review article by Gomez-Rios et al. 'Coated blade spray (CBS): shifting the paradigm of direct sample introduction to MS' published in January this year was picked up by several news outlets, the impact of which was evident through its Altmetrics score. The goal of the article was to concisely introduce CBS's operational 
fundamentals and to consider how it correlates/contrasts with existing direct-to-MS technologies suitable for bioanalytical applications [11].

\section{8 and beyond}

Bioanalysts are adapting continuously to advanced technologies, regulatory requirements and the likes, and Bioanalysis endeavours to capture the details and discussions of this continuously evolving field. We have a dedicated Editorial Board with experts from across the globe who play an important role in ensuring we publish the most up-to-date content, cover the hot topics and also maintain the high scientific standing of Bioanalysis. The timely scientific input from reviewers also plays a crucial role in this regard.

We are very thankful to the contribution of these experts in setting the tone and standard of the journal. We are delighted to have reached this milestone issue, knowing we do our utmost to stay in line with the developments of the field. Of course this would not be possible without the support and contributions from all our authors, readers, reviewers and editorial board members, all of with whom we would like to celebrate this occasion. We would like to thank everyone for your continued engagement and support, and here's to the next 200 issues!

\section{Financial \& competing interests disclosure}

$S$ Nadarajah is an employee of Future Science Ltd. The author has no other relevant affiliations or financial involvement with any organization or entity with a financial interest in or financial conflict with the subject matter or materials discussed in the manuscript apart from those disclosed.

No writing assistance was utilized in the production of this manuscript.

\section{References}

1. Bioanlaysis Zone. www.bioanalysis-zone.com

2. The Bioanalysis Glossary (2 ${ }^{\text {nd }}$ Edition). Future Science Group, London, UK

3. Hughes NC, Bajaj N, Fan J, Wong EYK. Assessing the matrix effects of hemolyzed samples in bioanalysis. Bioanalysis 1(6), 1057-1066 (2009).

4. Rangiah K, Hwang W-T, Mesaros C, Vachani A, Blair IA. Nicotine exposure and metabolizer phenotypes from analysis of urinary nicotine and its 15 metabolites by LC-MS. Bioanalysis 3(7), 745-761 (2011).

5. Theodorsson E. Validation and verification of measurement methods in clinical chemistry. Bioanalysis 4(3), 305-320 (2012).

6. Schwickart M, Vainshtein I, Lee R, Schneider A, Liang M. Interference in immunoassays to support therapeutic antibody development in preclinical and clinical studies. Bioanalysis 6(14), 1939-1951 (2014).

7. Spooner N, Cape S, Hayes R et al. Issues facing the bioanalytical community: summary of round table discussions. Bioanalysis 8(21), 2189-2193 (2016).

8. Zhang C, Bills BJ, Manicke NE. Rapid prototyping using 3D printing in bioanalytical research. Bioanalysis 9(4), 329-331 (2017).

9. Goodman J, Cowen S, Devanarayan V et al. Feedback from the European Bioanalysis Forum: focus workshop on current analysis of immunogenicity: best practices and regulatory hurdles. Bioanalysis 10(4), 197-204 (2018).

10. Garofolo W, Savoie N. The Decennial Index of the White Papers in Bioanalysis: 'A Decade of Recommendations (2007-2016)'. Bioanalysis 9(21), 1681-1704 (2017).

11. Gomez-Rıos GA, Tascon M, Pawliszyn J. Coated blade spray: shifting the paradigm of direct sample introduction to MS. Bioanalysis 10(4), 257-271 (2018). 
